\title{
Contribution à la connaissance des plantes médicinales utilisées dans les soins infantiles en pays San, au Burkina Faso
}

\section{P. ZERBO ${ }^{1 *}$, J. MILLOGO-RASOLODIMBY $^{1}$, O. G. NACOULMA-OUEDRAOGO ${ }^{2}$ et P. VAN DAMME ${ }^{3}$}

\footnotetext{
${ }^{1}$ Laboratoire de Biologie et Ecologie Végétales, Département de Biologie Végétale et Physiologie Végétale, Unité de Formation et de Recherche en Sciences de la Vie et de la Terre, Université de Ouagadougou, 03 BP 848 Ouagadougou 03, Burkina Faso.

${ }^{2}$ Laboratoire de Biochimie et de Chimie Appliquées (LABIOCA), Département de Chimie et Biochimie, Unité de Recherche et de Formation en Sciences de la Vie et de la Terre, Université de Ouagadougou, 03 BP 848 Ouagadougou 03, Burkina Faso.

${ }^{3}$ Laboratoire d'agronomie tropicale et subtropicale et d'ethnobotanique, Département de Production Végétale, Coupure links 653 B-9000, Université de Gent, Belgique.

*Auteur correspondant,E-mail: patrice_zerbo@univ-ouaga.bf, patzerbo@yahoo.fr
}

\begin{abstract}
RESUME
La présente étude contribue à la connaissance des plantes médicinales et des recettes médicamenteuses utilisées dans les soins infantiles au Pays San, situé au Nord-ouest du Burkina Faso. A la suite d'enquêtes ethnobotaniques, 50 tradipraticiens de santé, ayant au moins 15 ans d'expérience ont été interrogés. La technique utilisée était l'entretien semi-directif à partir d'un questionnaire élaboré. Au total 79 espèces médicinales ont été recensées, reparties en 69 genres et 34 familles dont les mieux représentées sont les Ceasalpiniaceae ( 7 genres et 9 espèces), les Mimosaceae ( 6 genres et 9 espèces), les Combretaceae (4 genres et 8 espèces) et les Anacardiaceae (4 genres et 5 espèces). Quelques $90 \%$ de ces espèces sont des plantes ligneuses (arbres, arbustes, lianes et sous-arbrisseaux). Isolées ou en association, ces plantes entrent dans la formulation de 167 recettes médicamenteuses, et les feuilles constituent les principaux organes sollicités. La décoction et la boisson sont prédominantes pour l'acquisition et l'administration des remèdes respectivement. Le paludisme et la diarrhée sont les plus cités sur les 34 maladies infantiles recensées.
\end{abstract}

(C) 2007 International Formulae Group. All rights reserved.

Mots clés: Plantes médicinales, Ethnopharmacopée, Pédiatrie traditionnelle, Burkina Faso.

\section{INTRODUCTION}

Dans de nombreux pays tropicaux et surtout en milieu rural, les structures sanitaires sont moins développées voire même inexistantes. De plus et malgré l'avènement des médicaments génériques, de nombreux traitements demeurent financièrement encore inaccessibles aux populations économiquement démunies. C'est ainsi qu'elles continuent à se tourner vers la nature pour $\mathrm{y}$ rechercher des remèdes essentiels à base de plantes. La médecine et la pharmacopée traditionnelles restent alors les seules sources de remède possible. Selon le CAPES (2004), 90\% de la population burkinabè ont actuellement recours à ces pratiques thérapeutiques. Pour documenter et ainsi pérenniser ce savoir traditionnel, divers travaux de recherches ethnopharmacologiques ont été entrepris. Certains d'eux ont couvert l'Afrique tropicale (Bartels, 1993), l'Afrique Sahélienne (Fortin et al., 1990), l'Afrique de l'Ouest (Daziel, 1937; Aké-Assi et Guinko, 1992) voire l'Afrique entière (Pousset, 1989; Sofowora, 1996). D'autres travaux ont porté sur des zones plus restreintes dans la sousrégion : en Côte d'Ivoire (Kerharo et Bouquet, 1950 ; Adjanohoun et al., 1979; Koné et al., 2002), au Mali (Adjanohoun et al., 1979; Togola et al., 2005), au Niger (Adjanohoun et al., 1980; Wezel, 2001), au Togo (Adjanohoun et al., 1986) ou au Bénin (Adjanohoun et al., 1989). 
Au Burkina Faso, certains travaux de recherches portant sur l'inventaire et l'utilisation des plantes médicinales ont été menés chez les Mossi du plateau central (Bognounou et al., 1975; NacoulmaOuédraogo, 1996), chez les Gourmantché à l'Est (Thiombiano et al., 2002), dans les jachères à l'Ouest (Olivier et Sanou, 2003) et chez les Goin (Ouôba et al., 2006) également à l'Ouest. D'autres travaux ont porté sur la commercialisation dans les marchés (Fernandez de la Pradilla, 1982 ; Lambert, 2003), etc. Cependant peu de travaux portent sur les plantes utilisées dans les soins des enfants. A ce titre, on peut citer les travaux de Flahaut (1999) sur les soins du couple mèreenfant, de Burgund (2002) sur des méthodes de renforcement de défenses naturelles chez le nourrisson et ceux de Nana-Sanon (2005) faisant l'inventaire des plantes médicinales utilisées dans les soins des enfants dans la commune de Ouagadougou.

Il reste cependant plusieurs régions et de nombreux groupes ethniques qui n'ont jamais été l'objet de travaux sur les plantes médicinales. C'est ainsi que nous avons entrepris une étude au «Pays San». L'objectif visé était de faire l'inventaire des plantes médicinales et des recettes médicamenteuses utilisées dans le traitement des maladies affectant les enfants d'âge inférieur à 14 ans.

Situé au Nord-ouest du Burkina Faso à environ $250 \mathrm{~km}$ de la capitale, le Pays San s'étend sur une superficie de $9691 \mathrm{~km}^{2}$ (Figure 1). Il est approximativement compris entre les longitudes $2^{\circ} 50^{\prime}$ et $3^{\circ} 20^{\prime}$ 'Ouest et les latitudes $12^{\circ} 40^{\prime}$ et $13^{\circ} 60^{\prime}$ Nord. Selon le découpage territorial, il correspond aux Provinces du Sourou et du Nayala (Ky, 1994). Au recensement général de la population et de l'habitat de 1996, on y dénombrait près de 325 000 habitants. Les groupes ethnolinguistiques en présence sont les Sana (45\%), les Dafing (37\%), les Mossi, les Peuls, les Nounouma, les Lélé, les Bobo et les Bwa (MEDEV, 2005). La végétation, de type arbustive, connaît une dégradation d'année en année. Cette zone est régie par un climat Soudanosahélien caractérisé par une longue période sèche (7-8 mois), une pluviométrie comprise entre 500 et $700 \mathrm{~mm}$ et des températures moyennes annuelles oscillant entre 17 et 22 ${ }^{\circ} \mathrm{C}$. Le vent dominant est l'harmattan (Fontes et Guinko, 1995). La végétation de type arbustive connaît une dégradation d'année en année (MECV, 2004).

\section{MATERIEL ET METHODES}

Cette étude a été réalisée à partir d'une série d'enquêtes ethnobotaniques utilisant l'entretien semi-directif à partir d'un questionnaire pré-élaboré. Les questions ont été posées dans la langue locale, le San. Au total 50 tradipraticiens de santé et herboristes ont été interrogés. Ils avaient tous une expérience d'au moins 15 ans dans l'utilisation des plantes en médecine et pharmacopée traditionnelles. L'échantillonnage était de type aléatoire dans les localités visitées. Le renseignement a porté sur les plantes qu'ils utilisent pour traiter les maladies, les organes de la plante, le mode de préparation des recettes et le mode d'administration. Lors des enquêtes, nous avons insisté sur les recettes concernant les maladies affectant les enfants. Trois (03) des enquêtés dont 2 infirmiers à la retraite et 1 chasseur (Dozo: Société traditionnelle de chasseurs, maître de la brousse), ont participé à l'identification des maladies et des plantes.

L'identification scientifique des espèces a été réalisée au Laboratoire de Biologie et Ecologie Végétales de l'Université de Ouagadougou où des espèces ont été comparées à celles de l'Herbier OUA. En outre la liste des espèces recensées dans la zone d'étude a été comparée avec celle du Catalogue des Plantes Vasculaires du Burkina Faso (Lebrun et al., 1991) et au lexique floristique proposé par Von Maydell (1983), Arbonnier (2000) et Bosch et al. (2002).

\section{RESULTATS}

Du tri réalisé, nous avons obtenu 79 plantes médicinales (Tableau 1) réparties en 69 genres et 34 familles (Tableau 2). Les plantes recensées sont composées de 32 arbres, 34 arbustes, 03 arbustes lianescents et lianes, 02 sous arbrisseaux vivaces par leurs rhizomes et 08 herbes annuelles (Tableau 3). Ces espèces interviennent dans la confection de 161 recettes médicamenteuses dont 27 sont des associations de 2 ou 3 plantes.

La fleur, le fruit, la feuille, l'écorce (tronc et racine), la racine et la plante entière sont les organes utilisés. Les feuilles ont une fréquence de citation de $41 \%$ et $25 \%$ pour l'écorce du tronc (Tableau 4). 


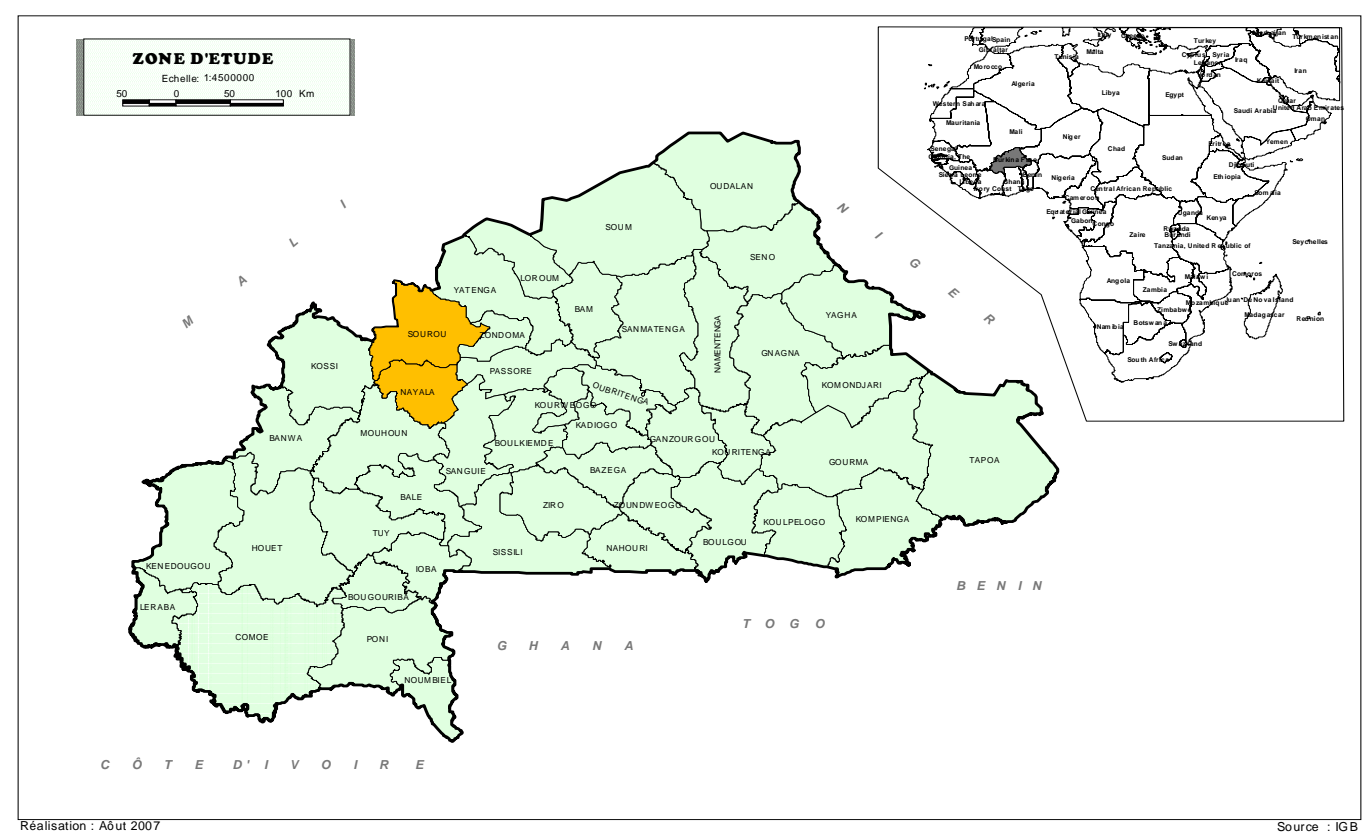

Figure 1: Zone d'étude, le Pays San : Provinces du Nayala et du Sourou.

(Source : Institut Géographique du Burkina, 2007)

La décoction, la macération aqueuse, l'infusion aqueuse et la calcination sont les modes de préparation des recettes. La boisson, le bain, le lavement, le massage, la fumigation, l'inhalation, l'instillation, l'application ou la scarification sont les modes d'administration des principes actifs.

Le paludisme et la diarrhée sont les principales maladies citées et traitées sur 34 maladies recensées. Vingt-quatre espèces appartenant à 14 familles sont utilisées dans le traitement du paludisme et 22 autres issues de 12 familles soignent la diarrhée.

\section{DISCUSSION}

Selon l'UNICEF (2004), près de 11 millions d'enfants meurent chaque année dans la plupart des pays tropicaux. Quelques $70 \%$ des décès sont dus au paludisme et à la diarrhée. Au Burkina Faso, ces deux maladies demeurent les premières causes de mortalité infantiles avec respectivement $12,50 \%$ et $15,60 \%$ de décès (MS, 2000).

Pour traiter le paludisme, diverses recettes sont élaborées. Les espèces utilisées ont des applications similaires dans d'autres régions du Burkina Faso et dans les pays avoisinants. Des espèces comme Sarcocephalus latifolius sont également utilisées par les Goins (Ouôba et al., 2006), Cochlospermum tinctorium, Combretum micranthum, Ozoroa insignis utilisées dans la commune de Ouagadougou (Nana-Sanon, 2005), par les Gourmatché (Thiombiano et al., 2002) et par les Mossi (Nacoulma-Ouédraogo, 1996). Selon ce dernier auteur, ces plantes renferment des pro-inhibiteurs que sont les alcaloïdes et les quinones. Ces métabolites réduisent ou stoppent complètement le développement in vitro des micro-organismes. Quant à la diarrhée, les espèces Adansonia digitata, Maytenus senegalensis, Acacia nilotica, Euphorbia hirta sont utilisées par les Sénoufo du Nord de la Côte d'Ivoire (Koné et al., 2002), par les populations à l'Ouest du Burkina Faso (Flahaut, 1999) et au plateau central (Nacoulma-Ouédraogo, 1996). Ces plantes renferment des tanins galliques qui inhibent la prolifération des germes pathogènes. 
P. ZERBO et al. / Int. J. Biol. Chem. Sci. 1(3): 262-274, 2007

Tableau 1 : Espèces médicinales recensées et leurs utilisations

\begin{tabular}{|c|c|c|c|c|c|c|}
\hline Familles & Genres et espèces & F. B. & $\begin{array}{l}\text { Nom local } \\
\text { en San }\end{array}$ & $\begin{array}{l}\text { Organes } \\
\text { utilisés }\end{array}$ & $\begin{array}{l}\text { Indications } \\
\text { thérapeutiques }\end{array}$ & Modes de préparation et d'administration \\
\hline \multirow{5}{*}{ Anacardaiceae } & Lannea acida A. Rich & A & Kaatô & $\begin{array}{l}\text { Tige feuillée } \\
\text { Ecorce-tronc ou Feuilles }\end{array}$ & $\begin{array}{l}\text { Diarrhée } \\
\text { Maux de ventre }\end{array}$ & $\begin{array}{l}\text { Décocté en boisson } \\
\text { Décocté en boisson }\end{array}$ \\
\hline & Lannea microcarpa Engl. et K. Krause & A & Tŏo & $\begin{array}{l}\text { Ecorce-racine et feuilles } \\
\text { Jeunes feuilles }\end{array}$ & $\begin{array}{l}\text { Paludisme } \\
\text { Diarrhée }\end{array}$ & $\begin{array}{l}\text { Décocté du mélange en boisson et bain } \\
\text { Filtrat du macéré en boisson }\end{array}$ \\
\hline & Mangifera indica $\mathrm{L}$. & A & Mange & Ecorce-tronc et Feuilles & Diarrhée & Décocté ou filtrat du macéré en boisson \\
\hline & Ozoroa insignis Del. & Arb & Korkân & $\begin{array}{l}\text { Ecorce-tronc et Feuilles } \\
\text { Feuilles } \\
\text { Racines } \\
\text { Ecorce-tronc }\end{array}$ & $\begin{array}{l}\text { Paludisme } \\
\text { Fièvre nocturne } \\
\text { Maux de ventre } \\
\text { Fissure anale }\end{array}$ & $\begin{array}{l}\text { Décocté en boisson et bain } \\
\text { Décocté ed l'organe avec les feuilles de Guiera senegalensis } \\
\text { en boisson } \\
\text { Décocté de l'organe avec les feuilles de Saba senegalensis et } \\
\text { lécorce-tronc de Sclerocarya birrea en boisson } \\
\text { Décocté en bain de siege }\end{array}$ \\
\hline & Sclerocarya birrea (A. Rich.) Hochst. & A & Sòrò & $\begin{array}{l}\text { Ecorce-tronc } \\
\text { Ecorce-tronc } \\
\text { Racines } \\
\text { Racines }\end{array}$ & $\begin{array}{l}\text { Toux } \\
\text { Paludisme } \\
\text { Maux de ventre } \\
\text { Dermatose }\end{array}$ & $\begin{array}{l}\text { Décocté en boisson } \\
\text { Décocté de l'organe avec l'écorce-tronc de Vitellaria } \\
\text { paradoxa en boisson } \\
\text { Filtrat de la poudre à l'eau en boisson } \\
\text { Décocté en bain }\end{array}$ \\
\hline Annonaceae & Annona senegalensis Pers. & Arb & Gini kuun & $\begin{array}{l}\text { Racines } \\
\text { Jeunes feuilles } \\
\text { Feuilles }\end{array}$ & $\begin{array}{l}\text { Diarrhée } \\
\text { Colique } \\
\text { Arrêt de la fontanelle }\end{array}$ & $\begin{array}{l}\text { Décocté en boisson } \\
\text { Décocté en boisson } \\
\text { Triturer les bourgeons et appliquer la pâte obtenue }\end{array}$ \\
\hline Apocynaceae & Saba senegalensis (A. DC) Pichon & Liane & Márà & \begin{tabular}{|l} 
Feuilles \\
Fruit
\end{tabular} & $\begin{array}{l}\text { Colique } \\
\text { Arrêt de la fontanelle }\end{array}$ & $\begin{array}{l}\text { Décocté en lavement } \\
\text { Application du mélange calcinat et beurre de Vitellaria } \\
\text { paradoxa }\end{array}$ \\
\hline Arecaceae & Borassus aethiopium Mart. & A & Sèwó & $\begin{array}{l}\text { Inflorescence } \\
\text { Bourgeons }\end{array}$ & $\begin{array}{l}\text { Teigne de la tête } \\
\text { Bilharziose }\end{array}$ & $\begin{array}{l}\text { Application du mélange calcinat et beurre de Vitellaria } \\
\text { paradoxa sur les parties atteintes. } \\
\text { Décocté en boisson }\end{array}$ \\
\hline \multirow[t]{2}{*}{ Asclepiadaceae } & Calotropis procera (Aiton) R. Br. & Arb & Semelefu & \begin{tabular}{|l} 
Tige feuillée \\
Ecorce-tronc
\end{tabular} & $\begin{array}{l}\text { Toux } \\
\text { Douleur générale }\end{array}$ & $\begin{array}{l}\text { Sucer la poudre du calcinat du rameau mort } \\
\text { Massage du corps avec le mélange de poudre au beurre de } \\
\text { Vitellaria paradoxa }\end{array}$ \\
\hline & Leptadenia hastata (Pers.) Decne & Liane & Giãkurun & Feuilles & Paludisme & Décocté en boisson \\
\hline \multirow[b]{2}{*}{ Asteraceae } & Chrysanthellum indicum DC. & $\mathrm{H}$ & Kárà gôn tisi & Ecorce-tronc & Fortifiant & Décocté en boisson \\
\hline & Vernonia colorata (Willd.) Drake & Arb & Kôôsafana & \begin{tabular}{|l} 
Feuilles \\
Feuilles
\end{tabular} & $\begin{array}{l}\text { Paludisme } \\
\text { Fièvre }\end{array}$ & $\begin{array}{l}\text { Décocté de l'organe avec les feuilles de Senna alata en } \\
\text { boisson et bain } \\
\text { Décocté de l'organe avec les feuilles de Senna alata en } \\
\text { boisson et bain }\end{array}$ \\
\hline Balanitaceae & Balanites aegyptiaca (L.) Del. & A & Semsle & \begin{tabular}{|l} 
Fruit \\
Ecorce-tronc
\end{tabular} & $\begin{array}{l}\text { Constipation } \\
\text { Paludisme }\end{array}$ & $\begin{array}{l}\text { Filtrat du macéré concentré en boisson } \\
\text { Décocté en boisson }\end{array}$ \\
\hline
\end{tabular}


P. ZERBO et al. / Int. J. Biol. Chem. Sci. 1(3): 262-274, 2007

\begin{tabular}{|c|c|c|c|c|c|c|}
\hline \multirow[t]{3}{*}{ Bombacaceae } & Adansonia digitata $\mathrm{L}$. & A & Kõñ & \begin{tabular}{|l} 
Ecorce-tronc \\
Ecorce-tronc \\
Pulpe séchée \\
Feuilles sèches
\end{tabular} & $\begin{array}{l}\text { Fièvre } \\
\text { Retard de croissance } \\
\text { Diarrhhée } \\
\text { Diarrhée }\end{array}$ & $\begin{array}{l}\text { Décocté en boisson } \\
\text { Décocté en bain } \\
\text { Filtrat du macéré en boisson } \\
\text { Consommation de lorgane avec les graines écrasées de } \\
\text { Hibiscus sabdariffa L. (Malvaceae) }\end{array}$ \\
\hline & Bombax costatum Pellegr. \& Vuillet & A & Berę & Gomme & Bilharziose & $\begin{array}{l}\text { Absorption de l'organe avec la bouillie du petit mil : } \\
\text { Pennisetum glaucum Staff et Hubb (Poaceae) }\end{array}$ \\
\hline & Ceiba pentandra (L.) Gaertn. & A & Jisè & Feuilles & Retard de croissance & Décocté en bain \\
\hline \multirow{9}{*}{ Caesalpinaceae } & Afzelia africana Smith ex Pers. & $\mathrm{A}$ & Tehiwii & Ecorce-tronc & Enfant chétif & Décocté du mélange en boisson \\
\hline & Cassia sieberiana DC. & Arb & Gisiĩ & $\begin{array}{l}\text { Feuilles } \\
\text { Feuilles et racines } \\
\text { Racines }\end{array}$ & $\begin{array}{l}\text { Enfant chétif } \\
\text { Boutons sur la peau } \\
\text { Maux de ventre }\end{array}$ & $\begin{array}{l}\text { Décocté en bain et fumigation } \\
\text { Décocté du mélange en boisson et bain } \\
\text { Filtrat de poudre et du jus de citron de Citrus aurantifolia } \\
\text { (Christm.) Swingle (Rutaceae) en boisson }\end{array}$ \\
\hline & Daniellia oliveri (Rolfe) Hutch. \& Dalz. & A & Gio & Tige feuillée & Douleur générale & Décocté en fumigation \\
\hline & Detarium microcarpa Guill et Perr. & A & Koro & $\begin{array}{l}\text { Ecorce-tronc } \\
\text { Ecorce-tronc } \\
\text { Ecorce-tronc } \\
\text { Fruit }\end{array}$ & $\begin{array}{l}\text { Fièvre } \\
\text { Paludisme } \\
\text { Fissure anale } \\
\text { Enfant chétif }\end{array}$ & $\begin{array}{l}\text { Massage avec la poudre et beurre de Vitellaria paradoxa } \\
\text { Décocté en bain } \\
\text { Filtrat de poudre à '’eau en bain du siège ou application de } \\
\text { poudre avec beurre de Vitellaria paradoxa en application } \\
\text { Pulpe du fruit à consommer }\end{array}$ \\
\hline & Piliostigma reticulatum (DC) Hochst & Arb & Gôntôrô & $\begin{array}{l}\text { Ecorce-tronc } \\
\text { Ecorce-tronc } \\
\text { Feuilles } \\
\text { Feuilles } \\
\text { Feuilles }\end{array}$ & \begin{tabular}{|l} 
Diarrhée \\
Dysenterie \\
Douleur générale \\
Paludisme \\
Bronchite ou toux
\end{tabular} & $\begin{array}{l}\text { Décocté en boisson } \\
\text { Décocté en boisson } \\
\text { Décocté en boisson et bain } \\
\text { Décocté de l'organe avec les feuilles de Guiera senegalensis } \\
\text { en boisson } \\
\text { Décocté de l'organe avec les feuilles de Guiera senegalensi en } \\
\text { boisson }\end{array}$ \\
\hline & Piliostigma thonningii (Schum.) Milne-Redh. & Arb & Gôntôrô wùsu & Feuilles & Mycose & $\begin{array}{l}\text { Décocté de l'organe avec les feuilles de Detarium microcarpa } \\
\text { en boisson }\end{array}$ \\
\hline & Senna alata (L.) Roxb. & Arb & Kassia & Feuilles & Paludisme & $\begin{array}{l}\text { Décocté de l'organe avec les feuilles de Carica papaya et de } \\
\text { celles de Vernonia colorata en boisson et bain }\end{array}$ \\
\hline & Senna occidentalis L. & Arb & Yarawe & Feuilles & Paludisme & $\begin{array}{l}\text { Décocté de l'organe avec les feuilles de Azadirachta indica en } \\
\text { boisson et bain }\end{array}$ \\
\hline & Tamarindus indica $\mathrm{L}$. & A & Too & $\begin{array}{l}\text { Feuilles } \\
\text { Ecorce-tronc } \\
\text { Fruit }\end{array}$ & $\begin{array}{l}\text { Varicelle } \\
\text { Conjonctivite } \\
\text { Constipation }\end{array}$ & $\begin{array}{l}\text { Décocté ou macéré en bain } \\
\text { Décocté en instillation et bain des yeux } \\
\text { Jus ou macéré du vieux fruit en boisson à jeun }\end{array}$ \\
\hline Capparidaceae & Capparis corymbosa $\mathrm{L}$. & Arb & Nyankabii & $\begin{array}{l}\text { Racines } \\
\text { Racines }\end{array}$ & $\begin{array}{l}\text { Colique } \\
\text { Douleur générale }\end{array}$ & $\begin{array}{l}\text { Décocté en boisson } \\
\text { Décocté en boisson }\end{array}$ \\
\hline Caricaceae & Carica papaya $\mathrm{L}$. & $\mathrm{A}$ & Nassankrù & Feuilles & Paludisme & Décocté en boisson \\
\hline Celastraceae & Maytenus senegalensis (L.) Exell. & Arb & Lakozza & $\begin{array}{l}\text { Racines et Feuilles } \\
\text { Feuilles }\end{array}$ & \begin{tabular}{|l} 
Maux de ventre \\
Paludisme
\end{tabular} & \begin{tabular}{|l} 
Décocté en boisson \\
Décocté en boisson
\end{tabular} \\
\hline Cochlospermaceae & Cochlospermum tinctorium Perr. Ex A. Rich. & $\mathrm{Hv}$ & Biriinpen & Racines séchées & Paludisme & Décocté en boisson \\
\hline
\end{tabular}


P. ZERBO et al. / Int. J. Biol. Chem. Sci. 1(3): 262-274, 2007

\begin{tabular}{|c|c|c|c|c|c|c|}
\hline & Anogeissus leiocarpus (DC.) Guill. \& Perr. & A & Lôô & \begin{tabular}{|l} 
Feuilles \\
Ecorce-tronc \\
Tige feuillée
\end{tabular} & $\begin{array}{l}\text { Diarrhée } \\
\text { Fortifiant } \\
\text { Fortifiant }\end{array}$ & $\begin{array}{l}\text { Décocté en boisson } \\
\text { Décocté en boisson } \\
\text { Décocté ou infusion en boisson }\end{array}$ \\
\hline & Combretum glutinosum Perr. ex DC. & Arb & Kikiz & Feuilles ou tige feuillée & Dysenterie & Décocté en boisson \\
\hline \multirow{6}{*}{ Combretaceae } & Combretum micranthum G. Don & Arb & Giaãn & \begin{tabular}{|l} 
Feuilles \\
Racines et Feuilles \\
Feuilles \\
Tige feuillée
\end{tabular} & $\begin{array}{l}\text { Fortifiant } \\
\text { Douleur générale } \\
\text { Diarrhée } \\
\text { Paludisme }\end{array}$ & $\begin{array}{l}\text { Décocté en boisson } \\
\text { Décocté en boisson et bain } \\
\text { Décocté en boisson } \\
\text { Décocté de l'organe avec la racine de Cochlospermum } \\
\text { tinctorium en boisson et bain }\end{array}$ \\
\hline & Combretum nigricans Lepr. ex Guill. et Perr. & Arb & Taã & $\begin{array}{l}\text { Racines } \\
\text { Ecorce-tronc }\end{array}$ & $\begin{array}{l}\text { Diarrhée } \\
\text { Troubles intestinaux }\end{array}$ & $\begin{array}{l}\text { Décocté en boisson } \\
\text { Décocté en boisson }\end{array}$ \\
\hline & Combretum paniculatum Vent. & Arb & Karangiibala & \begin{tabular}{|l|} 
Ecorce-tronc \\
Ecorce-racines \\
\end{tabular} & \begin{tabular}{|l|}
$\begin{array}{l}\text { Fissure anale } \\
\text { Paludisme }\end{array}$ \\
\end{tabular} & $\begin{array}{l}\text { Décocté chaud en lavement et bain du siège } \\
\text { Décocté en boisson et bain }\end{array}$ \\
\hline & Guiera senegalensis J. F. Gmel. & Arb & Baranfurun & $\begin{array}{l}\text { Fruit } \\
\text { Racines } \\
\text { Racines }\end{array}$ & $\begin{array}{l}\text { Diarrhée } \\
\text { Dysenterie } \\
\text { Paludisme }\end{array}$ & $\begin{array}{l}\text { Décocté en boisson } \\
\text { Décocté en lavement } \\
\text { Décocté en boisson et bain }\end{array}$ \\
\hline & Terminalia avicenioides Guill. et Perr. & Arb & Kỗo & $\begin{array}{l}\text { Feuilles } \\
\text { Racines } \\
\text { Racines }\end{array}$ & $\begin{array}{l}\text { Toux } \\
\text { Diarrhée } \\
\text { Dysenterie }\end{array}$ & $\begin{array}{l}\text { Filtrat du macéré en boisson } \\
\text { Décocté en boisson } \\
\text { Décocté en boisson }\end{array}$ \\
\hline & Terminalia macroptera Guill. et Perr. & A & Kôõ ti & \begin{tabular}{|l} 
Feuilles \\
Feuilles \\
Feuilles \\
Ecorce-tronc \\
Racines \\
Racines
\end{tabular} & $\begin{array}{l}\text { Colique } \\
\text { Fièvre } \\
\text { Paludisme } \\
\text { Plaie de bouche } \\
\text { Fortifiant } \\
\text { Courbature }\end{array}$ & $\begin{array}{l}\text { Décocté en boisson } \\
\text { Décocté en boisson } \\
\text { Décocté en boisson } \\
\text { Décocté en bain } \\
\text { Décocté en boisson } \\
\text { Décoction en boisson }\end{array}$ \\
\hline Cucurbitaceae & Lagenaria siceraria (Molina) Standl. & $\mathrm{H}$ & Pãn & Tige feuillée & Hoquet & Filtrat du calcinat avec l'eau en boisson \\
\hline Ebenaceae & Diospyros mespiliformis Hochst. ex A. DC & A & Nêmìc & \begin{tabular}{|l} 
Fruit vert \\
Feuilles \\
Feuilles jeunes rouges
\end{tabular} & \begin{tabular}{|l} 
Diarrhée \\
Conjonctivite \\
Maux d'oreilles
\end{tabular} & $\begin{array}{l}\text { Macéré en boisson } \\
\text { Filtrat du macéré en instillation dans les yeux } \\
\text { Décocté en instillation dans les oreilles }\end{array}$ \\
\hline Euphorbiaceae & Euphorbia hirta L. & $\mathrm{H}$ & Nââ yonu & Plante entière & Dysenterie & Décocté en boisson \\
\hline & Dalbergia melanoxylon Guill. et Perr. & Arb & Kersse & \begin{tabular}{|l} 
Feuilles \\
Racines \\
Ecorce-tronc
\end{tabular} & \begin{tabular}{|l} 
Diarrhée \\
Dysenterie \\
Bronchite
\end{tabular} & $\begin{array}{l}\text { Décocté en boisson } \\
\text { Décocté en boisson } \\
\text { Décocté en boisson }\end{array}$ \\
\hline Fabaceae & Indigofera nigritana Hook. F. & Sarbr & Gàrá & Feuilles & Paludisme & Décocté en boisson \\
\hline & Pterocarpus erinaceus Poir. & A & Diu & $\begin{array}{l}\text { Graine } \\
\text { Ecorce-tronc }\end{array}$ & $\begin{array}{l}\text { Paralysie musculaire } \\
\text { Diarrhée }\end{array}$ & $\begin{array}{l}\text { Application ou scarification du calcinat au beurre de } \\
\text { Vitellaria paradoxa } \\
\text { Décocté en boisson }\end{array}$ \\
\hline
\end{tabular}


P. ZERBO et al. / Int. J. Biol. Chem. Sci. 1(3): 262-274, 2007

\begin{tabular}{|c|c|c|c|c|c|c|}
\hline \multirow{3}{*}{ Meliaceae } & Azadirachta indica A. Juss. & A & Nímì & Feuilles & $\begin{array}{l}\text { Paludisme, } \\
\text { Maux de tête }\end{array}$ & $\begin{array}{l}\text { Décocté de l'organe avec les feuilles de Senna occidentalis en } \\
\text { fumigation et boisson } \\
\text { Décocté de l'organe avec les feuilles de Senna occidentalis en } \\
\text { fumigation et boisson }\end{array}$ \\
\hline & Khaya senegalensis (Desr.) A. Juss. & A & Mŭà & Ecorce-tronc & \begin{tabular}{|l|} 
Anémie, \\
Drépanocytose
\end{tabular} & $\begin{array}{l}\text { Décocté ou macéré en boisson et bain } \\
\text { Décocté ou macéré en boisson et bain }\end{array}$ \\
\hline & Trichilia emetica Vahl. & Arb & Lonloron & Ecorce-racine & Fissure anale & $\begin{array}{l}\text { Décocté en bain de siège ou mélange de poudre au beurre de } \\
\text { Vitellaria paradoxa en application }\end{array}$ \\
\hline \multirow{9}{*}{ Mimosaceae } & Acacia dudgeoni Craib ex Holl. & Arb & Baala & $\begin{array}{l}\text { Ecorce-tronc } \\
\text { Ecorce-tronc }\end{array}$ & \begin{tabular}{|l|} 
Diarrhée \\
Dysenterie
\end{tabular} & $\begin{array}{l}\text { Décocté en boisson } \\
\text { Décocté en boisson }\end{array}$ \\
\hline & Acacia macrostachya A. Rohb. ex DC. & Arb & Zama & Feuilles & Maux de ventre & Décocté en lavement \\
\hline & Acacia nilotica (L) Willd. ex Del. & Arb & Nyan tięn & $\begin{array}{l}\text { Ecorce-tronc } \\
\text { Racines } \\
\text { Graine }\end{array}$ & $\begin{array}{l}\text { Dysenterie } \\
\text { Ballonnement } \\
\text { Maux d'oreille }\end{array}$ & $\begin{array}{l}\text { Décocté en boisson et lavement } \\
\text { Filtrat de la poudre et l'eau en lavement } \\
\text { Infusion ou filtrat du macéré en instillation }\end{array}$ \\
\hline & Acacia seyal Del. & Arb & Nyan fuiĩn & $\begin{array}{l}\text { Gomme } \\
\text { Ecorce-tronc } \\
\text { Ecorce-tronc } \\
\text { Ecorce-tronc }\end{array}$ & \begin{tabular}{|l} 
Toux \\
Colique \\
Dysenterie \\
Conjonctivite
\end{tabular} & $\begin{array}{l}\text { Sirop de l'organe en boisson } \\
\text { Décocté en boisson } \\
\text { Décocté en boisson } \\
\text { Décocté en bain ou instillation }\end{array}$ \\
\hline & Dichrostachys cinerea (L.) Wight. et Arn. & Arb & Tiĩ & $\begin{array}{l}\text { Racines } \\
\text { Fruit }\end{array}$ & $\begin{array}{l}\text { Toux } \\
\text { Maux d'oreille }\end{array}$ & $\begin{array}{l}\text { Boire le décocté } \\
\text { Filtrat du macéré en instillation }\end{array}$ \\
\hline & Entada africana Guill et Perr. & Arb & Sèré mõnaa & \begin{tabular}{|l|} 
Ecorce-tronc \\
Ecorce-tronc \\
\end{tabular} & \begin{tabular}{|l|} 
Diarrhée vomissement \\
Toux
\end{tabular} & \begin{tabular}{|l} 
Filtrat de poudre et l'eau en boisson \\
Décocté en boisson et inhalation
\end{tabular} \\
\hline & Faidherbia albida (Del.) A. Chev. & A & Siâ & Ecorce-tronc & Toux longue (Coqueluche) & Décocté en boisson \\
\hline & Parkia biglobosa (Jacq.) R. ex G. Don. & A & Koe & \begin{tabular}{|l} 
Ecorce-tronc \\
Feuilles
\end{tabular} & $\begin{array}{l}\text { Diarrhée } \\
\text { Rougeole }\end{array}$ & $\begin{array}{l}\text { Décocté en lavement } \\
\text { Filtrat de la poudre et l'eau en application sur le corps durant } \\
\text { la maladie }\end{array}$ \\
\hline & Prosopis africana ( Guill. Perr. et Rich.) Taub. & Arb & Koron & \begin{tabular}{|l|} 
Feuilles \\
Racines et Feuilles \\
Racines \\
Ecorce-tronc \\
\end{tabular} & \begin{tabular}{|l|} 
Enfant chétif \\
Douleur générale \\
Dysenterie \\
Bronchite ou toux \\
\end{tabular} & \begin{tabular}{|l|} 
Décocté en bain \\
Décocté en boisson et bain \\
Décocté en boisson \\
Décocté en boisson
\end{tabular} \\
\hline \multirow{3}{*}{ Moraceae } & Ficus ingens (Miq.) Miq. & A & Nekomkièra & \begin{tabular}{|l|} 
Ecorce-tronc \\
Ecorce-tronc \\
Latex du tronc \\
\end{tabular} & \begin{tabular}{|l|} 
Diarrhée \\
Dysenterie \\
Douleur ombilicale \\
\end{tabular} & $\begin{array}{l}\text { Décocté en boisson et lavement } \\
\text { Décocté en boisson } \\
\text { Application du latex sur le nombril }\end{array}$ \\
\hline & Ficus platyphylla Del. & A & Goaran ticen & Ecorce-tronc & Maux de ventre & Décocté en boisson \\
\hline & Ficus sycomorus L. & A & Górò & $\begin{array}{l}\text { Ecorce-tronc } \\
\text { Ecorce-tronc }\end{array}$ & \begin{tabular}{|l} 
Diarrhée \\
Dysenterie
\end{tabular} & $\begin{array}{l}\text { Décocté en lavement } \\
\text { Décocté en boisson }\end{array}$ \\
\hline Moringaceae & Moringa oleifera Lam. & Arb & Basankoen & Feuilles & Rachitisme (os faible) & Filtrat du macéré en boisson \\
\hline \multirow[t]{2}{*}{ Myrtaceae } & Eucalyptus camaldulensis Dehnhardt & A & Ekalyptiisi & Feuilles & Paludisme ou Fièvre & $\begin{array}{l}\text { Décocté chaud de l'organe avec les feuilles de Carica papaya } \\
\text { et celles Senna occidentalis en boisson, fumigation et bain }\end{array}$ \\
\hline & Psidium guajava $\mathrm{L}$. & Arb & Goave & Feuilles & Diarrhée & Filtrat du macéré en boisson \\
\hline
\end{tabular}


P. ZERBO et al. / Int. J. Biol. Chem. Sci. 1(3): 262-274, 2007

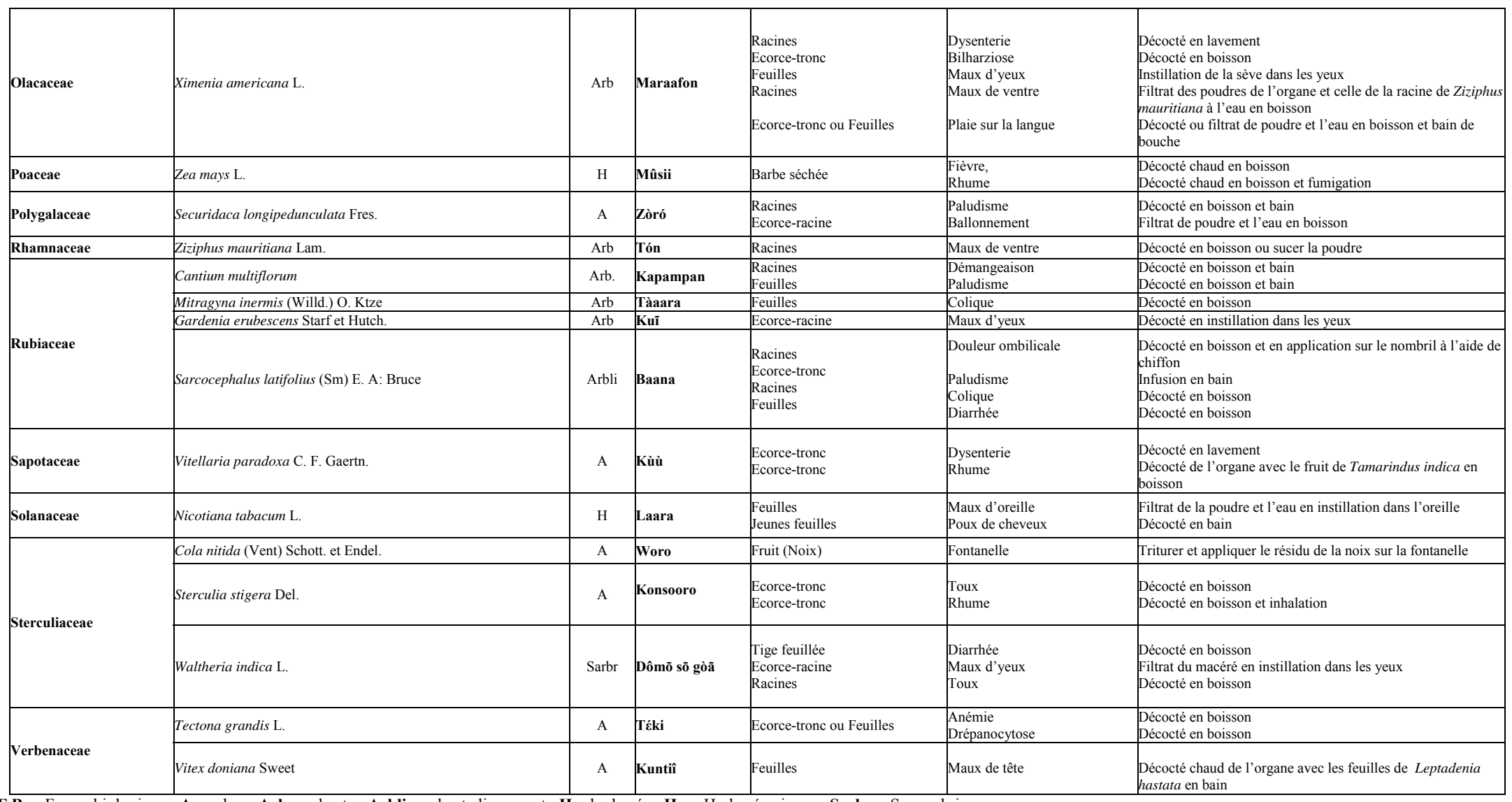

F.B. $=$ Forme biologique $; \mathbf{A}=$ arbre $; \mathbf{A r b}=$ arbuste $; \mathbf{A r b l i}=$ arbuste lianescent $; \mathbf{H}=$ herbacée $; \mathbf{H v}=$ Herbacée vivace $; \mathbf{S a r b r}=$ Sous arbrisseau

NB : Appellation San des espèces suivantes : Citrus aurantiflolia $($ Rutaceae $)=$ Lenburu ; Hibiscus sabdariffa $($ Malvaceae $)=$ Fõó ; Pennicetum glaucum $($ Poaceae $)=$ Nyànan. $($ Source : Données enquêtes 2004-2006) 
Tableau 2: Nombre de genres et d'espèces par famille

\begin{tabular}{lcc}
\hline Familles & $\begin{array}{c}\text { Nombre de } \\
\text { genres }\end{array}$ & $\begin{array}{c}\text { Nombre } \\
\text { d'espèces }\end{array}$ \\
\hline Ceasalpinaceae & 7 & 9 \\
Mimosaceae & 6 & 9 \\
Combretaceae & 4 & 8 \\
Anacardiaceae & 4 & 5 \\
Rubiaceae & 4 & 4 \\
Bombacaceae, Fabaceae, Meliaceae, Moraceae, Sterculiaceae & 3 & 3 \\
Asclepiadaceae, Asteraceae, Myrtaceae, Poaceae, Verbenaceae & 2 & 2 \\
\hline Annonaceae, Apocynaceae, Arecaceae, Balanitaceae, Capparidaceae, & & \\
Caricaceae, Celastraceae, Cochlospermaceae, Cucurbitaceae, Ebenaceae, & 1 & 1 \\
Euphorbiaceae, Malvaceae, Moringaceae, Olacaceae, Polygalaceae, & & \\
Rhamnaceae, Rutaceae, Sapotaceae, Solanaceae. & & $\mathbf{7 9}$ \\
\hline Total = 34 & $\mathbf{6 9}$ & \\
\hline
\end{tabular}

Tableau 3: Répartition des différentes formes biologiques

\begin{tabular}{|l|c|c|c|c|c|}
\hline Espèces & $\begin{array}{c}\text { Arbre } \\
\text { (A) }\end{array}$ & $\begin{array}{c}\text { Arbustes } \\
\text { (Arb) }\end{array}$ & $\begin{array}{c}\text { Autres ligneux } \\
\text { (Lianes Arbli + Sarbr) }\end{array}$ & $\begin{array}{c}\text { Herbacées } \\
(\mathbf{H}+\mathbf{H v})\end{array}$ & Total \\
\hline Nombre de citation & 32 & 34 & 05 & 8 & 79 \\
\hline Pourcentage & 41 & 43 & 06 & 10 & 100 \\
\hline
\end{tabular}

Tableau 4 : Fréquence de citation des organes.

\begin{tabular}{|l|c|c|c|c|c|}
\hline Organes & $\begin{array}{c}\text { Feuilles et } \\
\text { Tiges feuillées }\end{array}$ & Ecorce-tronc & $\begin{array}{c}\text { Racines et } \\
\text { écorces }\end{array}$ & Autres organes & Total \\
\hline Nombre de citation & 82 & 49 & 39 & 28 & 196 \\
\hline Pourcentage & 41 & 25 & 20 & 14 & 100 \\
\hline
\end{tabular}

Sept des 8 espèces de Combretaceae sont utilisées pour traiter le paludisme et la diarrhée. L'importance de cette famille floristique dans la vie de nos sociétés et en particulier dans la pharmacopée traditionnelle est bien connue (Bognounou, 1975 ; Nacoulma-Ouédraogo, 1996 ; Thiombiano et al., 2002). Selon ce dernier auteur, une trentaine de maladies sur 42 sont soignées exclusivement avec les Combretaceae ou en association avec des espèces d'autres familles dans la société Gourmantché (Burkina Faso). Quant aux Anacardiaceae, toutes les 5 espèces identifiées, interviennent dans les soins infantiles. Les vertus thérapeutiques des Anacardiaceae sont bien connues (Sérémé et al., 2001). Leur richesse en tanins serait à la base du traitement des diverses maladies.

Près de $90 \%$ d'espèces recensées sont des plantes ligneuses. Le caractère pérenne de ces espèces permettant de disposer d'au moins un organe en chaque saison pourrait justifier cette sollicitude. Olivier et Sanou (2003) de même que Nana-Sanon (2005) ont également constaté cette forte utilisation des espèces ligneuses en pharmacopée traditionnelle. Les feuilles sont plus utilisées dans le traitement des maladies infantiles (Flahaut, 1999) et les racines seraient réservées aux soins d'adultes Olivier et Sanou (2001). Cette préférence serait justifiée par l'idée séculaire des tradipraticiens selon laquelle « la racine, plus forte que la feuille doit soigner les adultes, eux-mêmes plus forts que les enfants, principalement soignés aux feuilles ». Pourtant Sourabié et al. (1995) ont montré que les feuilles de Sarcocephalus latifolius possèdent la même activité anti-diarrhéique que les racines. Selon Nacoulma-Ouédraogo (1996), ces organes périphériques sont les plus riches en métabolites secondaires et constituent les matériaux de base. Ces organes jouent un rôle protecteur et par conséquents utilisés dans la prévention des maladies infantiles (Burgund, 2002). 
Les résultats ont montré que certaines espèces à l'instar de Combretum glutinosum, Sarcocephalus latifolius, Waltheria indica peuvent traiter plus de deux maladies. Chez Combretum glutinosum, les feuilles sont utilisées pour soigner aussi bien le paludisme, la fièvre que les troubles gastriques (Adjanohoun et al., 1989; Pousset, 1989; Thiombiano, 1996). Les écorces du tronc de Sarcocephalus latifolius sont utilisées pour soigner les gastro-entérites infantiles, la dysenterie et le paludisme (Fernandez De la Pradilla, 1982 ; Ake-Assi et Guinko, 1992). Les racines de Waltheria indica sont utilisées pour lutter contre la fièvre (Olivier-Bever, 1986) et la toux sèche (Nacoulma-Ouédraogo, 1996). Ses tiges feuillées sont utilisées dans le traitement de la toux sèche et la diarrhée (Nacoulma-Ouédraogo, 1996). L'usage des plantes diffère donc selon la source d'information. Une comparaison de nos résultats avec ceux des travaux de Flahaut (1999), de Koné et al. (2002) ou de Nana (2005) montre peu de conformité de soins pour les mêmes maladies traitées par les mêmes espèces. Plusieurs auteurs ont également noté cette non conformité (Wezel, 2001; Ouôba et al., 2006). Cette situation témoigne de la complexité du domaine de la médecine et de la pharmacopée traditionnelles. En effet, les recettes et la connaissance des plantes qui entrent dans leur composition constituent des secrets qui ne sont confiés à une tierce personne que quand le dépositaire le veut et à qui il veut. Or selon Nacoulma-Ouédraogo (1996), les rituels de récoltes et les voies d'administration peuvent être considérés comme des trésors d'ingéniosité déployée pour optimiser la mise à la disposition des principes actifs.

\section{Conclusion}

Les résultats de nos enquêtes ont montré une grande diversité des espèces utilisées dans le traitement des maladies infantiles. Les 79 espèces identifiées sont réparties en 69 genres et 34 familles. Leurs actions médicales ont été vérifiées par plusieurs expériences traditionnelles à en croire ce proverbe africain : «l'arbre ne ment pas, c'est l'homme qui peut se tromper ». Des essais biologiques et chimiques permettront de vérifier la véracité des indications thérapeutiques reçues.
La diversité des propriétés et des indications, les divers organes utilisés ainsi que les modes de préparation et d'administration, témoignent d'une bonne connaissance de la diversité végétale des tradipraticiens de la zone d'étude.

Les feuilles, les écorces et les racines constituent les organes les mieux utilisés pour les soins des enfants. Après leur extraction, le problème de la survie des espèces se pose. Pour une meilleure utilisation des plantes médicinales, tout en préservant les ressources naturelles, une politique de sensibilisation des utilisateurs doit être élaborée en vue de leur enseigner les bonnes pratiques de récolte des plantes, la gestion et la protection des plantes in situ et les notions sur les techniques de culture des plantes médicinales. Pour réduire les risques de disparition, il est impératif d'entreprendre l'écologie des espèces très sollicitées. Ainsi les espèces rares ou en voie de disparition, trouveront une solution de survie par la régénération.

La complexité du domaine de la médecine traditionnelle a fait perdre des recettes suite à la disparition du détenteur du secret. Des études sur la connaissance des plantes médicinales d'autres ethnies sont à encourager afin d'archiver ces informations.

\section{REMERCIEMENTS}

La réalisation de ce travail a été possible grâce aux conseils et à la collaboration des chercheurs, des tradipraticiens de santé et herboristes suivants: Dr Ouétian Bognounou (Ethnobotaniste à la retraite, CNRST, Ouagadougou), Pasteur Elie So (alphabétiseur et transcripteur du san, langue parlée par les Sana), Mr Adama Sermé (reconnu par l'OAPI), Mr Jean Kéita (infirmier à la retraite), $\mathrm{Mr}$ Goumano Luc Zerbo, Mr Issiaka Péré Zerbo (Membre de la Confrérie des Dozo), Mr Siaka Ky (Tradipraticien-Herboriste), Mr Francis Paré (Institut Géographique du Burkina (IGB)). A tous, nous leur adressons nos sincères remerciements de même qu'à tous les tradipraticiens de santé et herboristes qui ont accepté partager leurs connaissances.

\section{REFERENCES BIBLIOGRAPHIQUES}

Adjanohoun EJ, Ake-Assi L. 1979. Contribution au recensement des plantes 
médicinales de la Côte d'Ivoire. Centre National de Floristique : Abidjan ; 359 p.

Adjanohoun EJ, Ake-Assi L, Floer JJ, Guinko S, Koumaré M, Ahyi AMR, Raynal JL. 1979. Médecine traditionnelle et pharmacopée - Contribution aux études ethnobotaniques et floristiques au Mali. ACCT : Paris ; 37-40.

Adjanohoun EJ, Ahyi AMR, Aké-Assi L, Dan Dicko L, Daouda H, Delams M, De Souza L, Garba M, Guinko S, Kagnonga A, N'Golo D, Raynal JL, Saadou M. 1980. Médecine traditionnelle et pharmacopée Contribution aux études ethnobotaniques et floristiques au Niger. ACCT: Paris; 58-67.

Adjanohoun EJ, Ahyi AMR, Aké-Assi L, Akpagana K, Chibon P, El-Adji A, Eymé J, Garba M, Gassita J-N, Gbeassor M, Goudoté E, Guinko S, Hodouto KK, Houngnon P, Kéita A, Kéoula Y, KlugaOcloo WP, Lo I, Siamevi KM, Taffamé KK. 1986. Médecine traditionnelle et pharmacopée - Contribution aux études ethnobotaniques et floristiques au Togo. ACCT : Paris; 122-135.

Adjanohoun EJ, Adjakidjé V, Aké-Assi L, Ahyi AMR, Akoegninou A, Almeida J, Apovo F, Boukef K, Chadaré M, Cusset G, Dramane K, Eymé J, Gassista J-N, Gbaguidi N, Goudoté E, Guinko S, Houngnon P, Keita A, Kiniffo HV, KonéBamba D, Musampa A, Saadou M, Sodogandji T, Souza De S, Tchabi A, Zinsou Dossa C, Zohoun T. 1989. Médecine traditionnelle et pharmacopée Contribution aux études ethnobotaniques et floristiques en République Populaire du Bénin. ACCT : Paris; 185-199.

Ake-Assi L, Guinko S. 1992. Plantes utilisées dans la médecine traditionnelle en Afrique de l'Ouest. Editions Roche-Basel: Switzerland ; 65-77.

Arbonnier M. 2000. Arbres, arbustes et lianes des zones sèches d'Afrique de l'ouest. CIRAD: Paris ; 541p.

Bartels A. 1993. Guide des plantes tropicales - Plantes ornementales, plantes utiles, fruits toxiques. Editions Eugen Ulmer; $384 \mathrm{p}$.

Bognounou O. 1975. Note sur une plante médicinale: le randga ou kinkeliba
(Combretum micranthum G. Don. Combretaceae). Bulletin Trimestriel Informations Scientifiques. Notes et Documents Voltaïques, 8(4): 36-44.

Bognounou $\mathrm{O}$, Ouédraogo $\mathrm{CO}$, Ouédraogo OG. 1975. Contribution à l'inventaire des plantes médicinales africaines en pays Mossi (Région de Ouagadougou). Bulletin Trimestriel Informations Scientifiques. Notes et Documents Voltaïques, 8(4): 50-56.

Bosch CH, Siemonsma JS, Lemmens RHM, Oyen LPA. 2002. Ressources végétales de l'Afrique Tropicale : listes de base des espèces et de leurs groupes d'usage. PROTA Programme Wageningen: The Netherlands ; 314p.

Burgund M. 2002. Etude des méthodes de renforcement de défenses naturelles chez le nourrisson au Burkina Faso. Mémoire de fin d'études de Doctorat en Pharmacie, Université Libre de Belgique, 65p.

CAPES (Centre d'Analyse des Politiques Economiques et Sociales). 2004. Analyse situationnelle dans quatre regions $d u$ Burkina Faso: Cascades, Hauts-Bassins, Sahel et Sud-Ouest. Rapport final. CAPES ; $241 \mathrm{p}$.

Daziel JM. 1937. The useful plants of West Africa: Appendix to the flora of West Tropical Africa. The Crown Agents for the Colonies: London; 612p.

Fernandez De la Pradilla C. 1982. Plantes médicinales vendues sur les marchés de Ouagadougou, Pabré Burkina Faso. $2^{\text {ème }}$ édition. Librairie Jeunesse d'Afrique; $32 \mathrm{p}$.

Flahaut E. 1999. Pharmacopée et médecine traditionnelle dans l'ouest du Burkina Faso. Plantes médicinales et soins du couple mère-enfant. Thèse de Doctorat en Pharmacie, Université de Lille II, 143p.

Fontes J, Guinko S. 1995. Notion de la carte de la végétation et l'occupation du sol du Burkina Faso. Ministère de la Coopération Française, projet CAMPUS (88 313 101), 59p.

Fortin D, Lo M, Maynart G. 1990. Plantes médicinales du sahel. Editions CECI/ENDA ; 300p.

Kerharo J, Bouquet A. 1950. Plantes médicinales et toxiques de la Côte 
d'Ivoire-Haute Volta. Editions Vigot: Paris ; 250p.

Koné MW, Atindehou KK, Téré H, Traoré D. 2002. Quelques plantes médicinales utilisées en pédiatrie traditionnelle dans la région de Férékessédougou, (Côte d'Ivoire). Actes du colloque international, Centre Suisse du 26-27 Août 2001. BIOTERRE, Revue Internationale des Sciences de la Vie et de la Terre, $n^{\circ}$ spécial, 2002. Editions Universitaires de Côte d'Ivoire, $30: 30-36$

Ky JC. 1994. Des masques du pays San (Burkina Faso) : Recherche des origines à travers l'histoire, le culte et l'art. Thèse de Doctorat, Université de Paris I, Pantheon-Sorbone, 514p.

Lambert JDH. 2003. Vente et commercialisation des plantes médicinales à Ouagadougou et BoboDioulasso, Burkina Faso : Evaluation socio-économique. Canadian Trust Funds ; $45 \mathrm{p}+$ annexes.

Lebrun JP, Toutain B, Gaston A, Boudet G. 1991. Catalogues des plantes vasculaires du Burkina Faso. Institut d'Elevage et de Médecine Vétérinaire des Pays Tropicaux, (Maisons Alfort), 341p.

MECV (Ministère de l'Environnement et du Cadre de Vie). 2004. Audit environnemental de l'Autorité de Mise en Valeur de la Vallée du Sourou. MECV; $89 \mathrm{p}$.

MEDEV (Ministère de l'Economie et du Développement). 2005. Profil des régions du Burkina Faso. MEDEV; 281p.

MS (Ministère de la Santé). 2000. Document d'analyse de la situation sanitaire nationale. Direction Etude et Planification, MS ; 120p.

Nacoulma-Ouédraogo OG. 1996. Plantes médicinales et Pratiques médicales traditionnelles au Burkina Faso : cas du plateau central. Thèse de Doctorat d'Etat ès Sciences Naturelles, Université de Ouagadougou, tome II, 285p.

Nana-Sanon P. 2005. Plantes médicinales utilisées pour les soins des enfants dans la commune de Ouagadougou : inventaire et culture de quelques espèces. Mémoire de fin d'études d'Ingénieur des Eaux et Forêts. $75 \mathrm{p}$.
Olivier-Bever B. 1986. Medicinal plants in tropical West Africa. Cambridge University Press ; 90-91.

Olivier M, Sanou L. 2001. Rôle des femmes et des tradipraticiens dans la conservation et la valorisation de la biodiversité dans le sud-ouest du Burkina Faso. In Pratiques culturelles, la sauvegarde et la conservation de la biodiversité en Afrique de l'Ouest et du Centre. Actes du séminaire-Atelier, Ouagadougou (Burkina Faso), 18 au 21 Juin ; 61-75.

Olivier M, Sanou L. 2003. Contribution à l'étude des plantes médicinales des jachères de l'ouest du Burkina Faso. Ethnopharmacologia, 30: 47-59.

Ouôba P, Lykke AM, Boussim J, Guinko S. 2006. La flore médicinale de la Forêt classée de Niangoloko (Burkina Faso). Etudes sur la flore et la végétation du Burkina Faso et des pays avoisinants. Vol. 10. Editions Verlag Natur \& Wissenschaft, Solingen : Francfort et Ouagadougou; 5-12.

Pousset JL. 1989. Plantes médicinales africaines - Utilisation pratique. Ellipses, ACCT : Paris ; 66-86.

Sereme A, Millogo-Rasolodimby J, KoudaBonafos M, Guinko S, Nacro M. 2001. Les vertus thérapeutiques des Anacardiaceae en liaison avec leurs métabolites notamment les tanins. Annale de Botanique de l'Afrique de l'Ouest, 00(0): 63-71.

Sofowora A. 1996. Plantes médicinales et médecine traditionnelle d'Afrique. $2^{\text {ème }}$ édition, Editions Karthala : Paris, France ; $378 \mathrm{p}$.

Sourabié S, Guinko S, Kaboré IZ. 1995. Contribution a l'étude chimique et microbiologique de Nauclea latfolia Sm. (Rubiaceae) : possibilités d'utilisation des feuilles comme succédané des racines dans le traitement traditionnel de gastroentérites. Revue de Médecine et Pharmacopée Africaine, 9(1): 7-12.

Thiombiano A. 1996. Contribuion à l'étude des Combretaceae dans les formations végétales de la région Est du Burkina Faso. Thèse de Doctorat de 3ème cycle des Sciences Biologiques Appliquées, Université de Ouagadougou, 220p. 
Thiombiano A, Ouôba P, Guinko S. 2002. Place des Combretaceae dans la société gourmantché à l'est du Burkina Faso. Etudes floristiques de la végétation du Burkina Faso et pays avoisinants, 7: 1722.

Togola A, Diallo D, Dembélé S, Barsett H, Smestad Paulsen B. 2005. Ethnopharmacological survey of different uses of seven medicinal plants from Mali, (West Africa) in the regions Doila, Kolokani and Siby. Journal of Ethnobiology and Ethnomedicine, 1:7.
UNICEF, 2004. Le paludisme : une cause majeure de mortalité infantile et de pauvreté en Afrique. Edittion UNICEF ; $17 \mathrm{p}$.

Von Maydell H-J. 1983. Arbres et arbustes du sahel: leurs caractéristiques et leurs utilisations. GTZ; 531p.

Wezel A. 2001. Plantes médicinales et leur utilisation traditionnelle chez les paysans au Niger. Etudes sur la flore et la végétation du Burkina Faso et des pays avoisinants. Vol. 6. Editions Verlag Natur \& Wissenschaft, Solingen: Francfort et Ouagadougou ; 9-18. 